\title{
Can contrast harmonic endoscopic ultrasonography replace endoscopic ultrasonography-guided fine-needle aspiration in patients with solid pancreatic lesions? An American perspective
}

\author{
Joseph Yoo, Linda H. Yan, Ali A. Siddiqui \\ Department of Internal Medicine, Division of Gastroenterology and Hepatology, Thomas Jefferson University Hospital, \\ Philadelphia, PA, USA
}

\section{INTRODUCTION}

Contrast harmonic endoscopic ultrasonography (CH-EUS) is a novel form of endoscopic ultrasonography (EUS) that utilizes intravenous contrast agents to characterize the vasculature inside an organ of interest with a broadband transducer that can detect harmonic signals. ${ }^{[1,2]}$ These contrast agents initially played a major role in echocardiography, enhancing the power of ultrasonography to image cardiac chambers and the nearby large blood vessels. ${ }^{[3]}$ They have been since utilized with EUS to aid clinicians in differentiating between benign and malignant lesions, in determining the depth of esophageal, gastric, and gallbladder cancer invasion, and in imaging the portal venous system and varices. ${ }^{[2]}$ Contrast agents are especially important in tumor imaging because tumor angiogenesis usually significantly alters the vasculature within the malignant lesion. The intravenous contrast agents are typically administered via an intravenous bolus injection of gaseous microbubbles that do not leave the vascular system and reflect the ultrasound waves. The contrast can also be injected as a

\begin{tabular}{|l|l|}
\hline \multicolumn{3}{|c|}{ Access this article online } \\
\hline Quick Response Code: & Website: \\
\hline & www.eusjournal.com \\
\cline { 2 - 2 } & \\
\hline
\end{tabular}

continuous infusion, which can alter the appearance of vasculature on CH-EUS due to the difference in intravascular density of the contrast material.

In the USA, accurately diagnosing solid lesions of the pancreas remains an area of ongoing study because it has significant therapeutic implications. Pancreatic ductal adenocarcinoma, the most common solid pancreatic tumor, has a very poor prognosis and often requires early surgical intervention to prevent rapid progression. Conversely, neuroendocrine tumors have a much slower rate of progression and do not typically require immediate surgical intervention. Inflammatory masses associated with chronic pancreatitis also usually do not require surgery and can be managed conservatively. CH-EUS has been proposed as an important imaging modality that can aid in distinguishing between the different types of pancreatic lesions, thereby potentially sparing patients from the high morbidity and mortality associated with pancreatic surgery. ${ }^{[4]}$

\footnotetext{
This is an open access article distributed under the terms of the Creative Commons Attribution-NonCommercial-ShareAlike 3.0 License, which allows others to remix, tweak, and build upon the work non-commercially, as long as the author is credited and the new creations are licensed under the identical terms.
}

How to cite this article: Yoo J, Yan LH, SiddiquiAA. Can contrastharmonic endoscopic ultrasonography replace endoscopic ultrasonography-guided fine-needle aspiration in patients with solid pancreatic lesions? An American perspective. Endosc Ultrasound 2017;6:1-3.

\section{Address for correspondence}

Dr. Ali A. Siddiqui, Thomas Jefferson University Hospital, Philadelphia, PA, USA. E-mail: ali.siddiqui@jefferson.edu

Received: 2016-02-15; Accepted: 2016-06-01 
Yoo, et al:: Can CH-EUS replace EUS-FNA?

\section{CONTRAST HARMONIC ENDOSCOPIC ULTRASONOGRAPHY: CURRENT DATA}

The current gold standard for the diagnosis of malignant pancreatic lesions, and for the differentiation between pancreatic tumors and chronic pancreatitis, is EUS-guided fine-needle aspiration (EUS-FNA) ${ }^{[5]}$ It has been well established in the literature that differentiating between pancreatic masses caused by inflammation and carcinoma can be challenging. Despite the fact that histopathologic evaluation is the gold standard for diagnosing pancreatic masses, it is not always accurate, as many carcinomas are surrounded by inflammatory changes. The surrounding inflammation has been shown to result in false-negative results, even when the tissue is obtained via EUS-FNA. ${ }^{[6]}$ As such, clinicians have continued to search for the best method for diagnosing pancreatic masses.

CH-EUS has been shown to be useful in pancreatic imaging by defining vascular landmarks, identifying the obliteration of vasculature by a thrombus or tumor, and by determining microvascular blood flow in the pancreas and pancreatic lesions. Current literature suggests that the degree of enhancement with contrast agents may be related to the amount of microcirculation and vascular permeability within a pancreatic lesion or to the degree of inflammation in pancreatic parenchyma. ${ }^{[7-11]}$

Normal pancreatic parenchyma and the areas of fibrosis typically enhance with contrast, while the appearance of pancreatic masses with CH-EUS depends on the cytopathology of the lesion. ${ }^{[7,8]}$ Pancreatic islet cell tumors and mucin-producing tumors typically have marked enhancement with contrast utilization, while pancreatic ductal cell carcinomas and pancreatic pseudocysts typically remain unenhanced due to their relative avascularity. ${ }^{[1]}$ Hirooka et al. demonstrated that these findings were consistent with the vascularity demonstrated by angiography except in $20 \%$ of the cases of mucin-producing tumors and in $25 \%$ of the cases of chronic pancreatitis. ${ }^{[1,7]}$ They also indicated that the boundaries of normal pancreatic parenchyma and fibrosis can be enhanced with contrast, helping to further delineate the pancreatic lesions. ${ }^{[7,8]}$ In addition, CH-EUS can be used to differentiate between mass-forming chronic pancreatitis and pancreatic cancer. Dietrich et al. demonstrated that the difference in tissue microperfusion between chronic pancreatitis and pancreatic cancer results in differences in appearance on CH-EUS. ${ }^{[10]}$ Whereas focal lesions due to chronic pancreatitis enhance inversely proportional to the degree of fibrosis and duration of inflammation within the mass, ductal carcinomas displayed a low level, or complete absence of enhancement, due to the relatively greater degree of fibrosis..$^{[1,10,12,13]}$

Taking advantage of the different appearances of varying pancreatic mass lesions on $\mathrm{CH}$-EUS has been shown to be quite effective in several studies in the international community. Based on the meta-analysis by Gong et al., CH-EUS has a great potential for differentiating pancreatic adenocarcinomas from other pancreatic masses, with a sensitivity of $94 \%(95 \%$ confidence interval [CI], 0.91-0.95) and a specificity of $89 \%(95 \% \mathrm{CI}, 0.85-0.92) .{ }^{[14]}$ In fact, multiple studies have shown that the negative predictive value for the diagnosis of adenocarcinoma in pancreatic masses is greater with CH-EUS than with EUS-FNA, and that $\mathrm{CH}$-EUS is more sensitive if not more than EUS-FNA. ${ }^{[15,16]}$

\section{AN AMERICAN PERSPECTIVE}

As previously discussed, multiple studies have demonstrated the advantages of using contrast-enhanced endoscopic ultrasound in the diagnosis of pancreatic mass lesions. The majority of these studies, however, continue to be primarily from Europe and Asia. In fact, the European Federation of Societies for Ultrasound in Medicine and Biology has already released updated clinical guidelines regarding the use of contrast-enhanced endoscopic ultrasound, with sections pertaining specifically to the pancreas. ${ }^{[17]}$

One of the earliest investigations into the potential of contrast-enhanced endoscopic ultrasound in North America was published by Bhutani et al. in 1997. ${ }^{[18]}$ These early experiments, performed on three 20-25 kg swine (Sus scrofa), demonstrated visually noticeable enhancement of the color Doppler signals from the celiac and superior mesenteric arteries after injections of SHU508 (Levovist). Based on their findings, Bhutani et al. hypothesized that vascular contrast agents had the potential to have a significant role in improving the diagnostic accuracy of pancreatic masses, as the celiac and superior mesenteric arteries serve as the two main sources of blood supply to the pancreas. ${ }^{[18]}$ Since these early experiments, however, the relative majority of studies utilizing contrast-enhanced endoscopic ultrasound have come from Europe and Asia. 
Yoo, et al:: Can $\mathrm{CH}-\mathrm{EUS}$ replace EUS-FNA?

In the previously mentioned meta-analysis by Gong et al., only one of the studies included was performed in the USA. ${ }^{[14]}$ The results of that study, by Romagnuolo et al., revealed a positive/negative predictive value of $80.0 \%$ (95\% CI, $51.9 \%-95.7 \%$ ) $/ 100.0 \%$ (95\% CI, 63.0\%-100.0\%) for contrast-enhanced harmonic EUS versus $84.6 \% / 100.0 \%$ for EUS in 24 cases with confirmed diagnoses (12 malignant and 12 benign). ${ }^{[19]}$ The conclusions from that study were consistent with the conclusions of the international community thus far; contrast-enhanced endoscopic ultrasound is safe, time-efficient, cost-conscious, and effective in the evaluation and diagnosis of pancreatic mass lesions when compared to the gold standard EUS-FNA. Unfortunately, publishing more American-based studies has proven to be difficult given the regulatory barriers that have been put in place by the Food and Drug Administration (FDA). ${ }^{[18]}$ Not only the use of ultrasound contrast agents has not been approved in the setting of endoscopic ultrasound, but also black-box warnings and contraindications issued by the FDA make it necessary to exercise a considerable amount of caution.

Currently, more studies need to be conducted in American centers before one can form a unique American perspective regarding the potential of $\mathrm{CH}-\mathrm{EUS}$ to replace EUS-FNA. EUS-FNA is the current gold standard, and as such, has been proven to be an effective method by which to diagnose solid pancreatic tumors and to differentiate malignancy from chronic pancreatitis. However, the aforementioned risk of obtaining falsely negative histopathology due to sampling tissue from the areas of surrounding inflammation as opposed to areas with malignant changes remains a major concern. One can argue that it is in these settings that CH-EUS has its greatest utility, not as a substitute to EUS-FNA, but as a complementary modality that can increase the diagnostic accuracy of EUS-FNA by identifying hypoenhancing regions to target for sampling. Furthermore, data released by Fusaroli and Eloubeidi and Gincul et al. have already lent support to this notion. ${ }^{[20-22]}$ Nevertheless, the final verdict regarding the role of CH-EUS, whether it acts as a complement or replacement, remains to be seen. From an American perspective, the first step toward obtaining the answer requires a regulatory shift toward more reasonable limitations pertaining to the use of contrast-enhancing agents so that further studies can be done.

\section{REFERENCES}

1. Reddy NK, Ioncica AM, Saftoiu A, et al. Contrast-enhanced endoscopic ultrasonography. World J Gastroenterol 2011;17:42-8.

2. Kitano M, Takagi $\mathrm{T}$, Sakamoto $\mathrm{H}$, et al. Dynamic imaging of pancreatic tumors by contrast-enhanced harmonic EUS with long-lasting contrast. Gastrointest Endosc 2009;69:S228.

3. Keller MW, Feinstein SB, Watson DD. Successful left ventricular opacification following peripheral venous injection of sonicated contrast agent: An experimental evaluation. Am Heart J 1987;114:570-5.

4. Carter DC. Cancer of the head of pancreas or chronic pancreatitis: Diagnostic dilemma with significant consequences. World J Surg 2003;27:1249-57.

5. Ryozawa S, Kitoh H, Gondo T, et al. Usefulness of endoscopic ultrasound-guided fine-needle aspiration biopsy for the diagnosis of pancreatic cancer. J Gastroenterol 2005;40:907-11.

6. Bhutani MS, Hawes RH, Baron PL, et al. Endoscopic ultrasound guided fine needle aspiration of malignant pancreatic lesions. Endoscopy 1997;29:854-8.

7. Hirooka $Y$, Naitoh $Y$, Goto $H$, et al. Usefulness of contrast-enhanced endoscopic ultrasonography with intravenous injection of sonicated serum albumin. Gastrointest Endosc 1997;46:166-9.

8. Hirooka Y, Goto H, Ito A, et al. Contrast-enhanced endoscopic ultrasonography in pancreatic diseases: A preliminary study. Am J Gastroenterol 1998;93:632-5.

9. Hyodo N, Hyodo T. Ultrasonographic evaluation in patients with autoimmune-related pancreatitis. J Gastroenterol 2003;38:1155-61.

10. Dietrich CF, Ignee A, Frey H. Contrast-enhanced endoscopic ultrasound with low mechanical index: A new technique. Z Gastroenterol 2005;43:1219-23.

11. Wong RF, Tarcin O, Reddy N, et al. Contrast-enhanced EUS (CE-EUS) using a new microbubble contrast agent (MRX-815) - A feasibility study in a porcine model. Gastrointest Endosc 2006;63:AB274.

12. Zhu Q. Study on contrast enhanced EUS in differential diagnosis of pancreatic tumor. Gastrointest Endosc 2009;69:S265.

13. D'Onofrio M, Zamboni G, Tognolini A, et al. Mass-forming pancreatitis: Value of contrast-enhanced ultrasonography. World J Gastroenterol 2006;12:4181-4.

14. Gong TT, Hu DM, Zhu Q. Contrast-enhanced EUS for differential diagnosis of pancreatic mass lesions: A meta-analysis. Gastrointest Endosc 2012;76:301-9.

15. Napoleon B, Alvarez-Sanchez MV, Gincoul R, et al. Contrast-enhanced harmonic endoscopic ultrasound in solid lesions of the pancreas: Results of a pilot study. Endoscopy 2010;42:564-70.

16. Kitano M, Kudo M, Yamao K, et al. Characterization of small solid tumors in the pancreas: The value of contrast-enhanced harmonic endoscopic ultrasonography. Am J Gastroenterol 2012;107:303-10.

17. Piscaglia F, Nolsøe C, Dietrich C, et al. The EFSUMB guidelines and recommendations on the clinical practice of contrast enhanced ultrasound (CEUS): Update 2011 on non-hepatic applications. Ultraschall Med 2012;33:33-59.

18. Bhutani MS, Hoffman BJ, van Velse A, et al. Contrast-enhanced endoscopic ultrasonography with galactose microparticles: SHU508 A (Levovist). Endoscopy 1997;29:635-9.

19. Romagnuolo J, Hoffman B, Vela S, et al. Accuracy of contrast-enhanced harmonic EUS with a second-generation perflutren lipid microsphere contrast agent (with video). Gastrointest Endosc 2011;73:52-63.

20. Fusaroli P, Spada A, Mancino MG, et al. Contrast harmonic echo-endoscopic ultrasound improves accuracy in diagnosis of solid pancreatic masses. Clin Gastroenterol Hepatol 2010;8:629-34.e1-2.

21. Fusaroli P, Eloubeidi MA. Diagnosis of pancreatic cancer by contrast-harmonic endoscopic ultrasound (EUS): Complementary and not competitive with EUS-guided fine-needle aspiration. Endoscopy 2014;46:380-1.

22. Gincul R, Palazzo M, Pujol B, et al. Contrast-harmonic endoscopic ultrasound for the diagnosis of pancreatic adenocarcinoma: A prospective multicenter trial. Endoscopy 2014;46:373-9. 